\title{
Priming global and local processing of composite faces: revisiting the processing-bias effect on face perception
}

\author{
Zaifeng Gao • Anastasia V. Flevaris • \\ Lynn C. Robertson • Shlomo Bentin
}

Published online: 26 February 2011

(C) The Author(s) 2011. This article is published with open access at Springerlink.com

\begin{abstract}
We used the composite-face illusion and Navon stimuli to determine the consequences of priming local or global processing on subsequent face recognition. The composite-face illusion reflects the difficulty of ignoring the task-irrelevant half-face while attending the task-relevant half if the half-faces in the composite are aligned. On each trial, participants first matched two Navon stimuli, attending to either the global or the local level, and then matched the upper halves of two composite faces presented sequentially. Global processing of Navon stimuli increased the sensitivity to incongruence between the upper and the lower halves of the composite face, relative to a baseline in which the composite faces were not primed. Local processing of Navon stimuli did not influence the sensitivity to incongruence. Although incongruence induced a bias toward different responses, this bias was not modulated by priming. We conclude that global processing of Navon stimuli augments holistic processing of the face.
\end{abstract}

Electronic supplementary material The online version of this article (doi:10.3758/s13414-011-0109-7) contains supplementary material, which is available to authorized users.

Z. Gao $\cdot$ S. Bentin

Department of Psychology, Hebrew University of Jerusalem,

Jerusalem 91905, Israel

\section{S. Bentin $(\bowtie)$}

Interdisciplinary Center for Neural Computation,

Hebrew University,

Jerusalem, Israel

e-mail: shlomo.bentin@huji.ac.il

\section{A. V. Flevaris $\cdot$ L. C. Robertson}

Veterans Administration Research, Martinez, CA, and Department of Psychology, University of California Berkeley, Berkeley, CA, USA
Keywords Face perception - Navon letter $\cdot$ Composite-face illusion $\cdot$ Holistic perception $\cdot$ Global processing $\cdot$ Local processing

Since the early days of the Gestalt school, the relative roles of the whole and its parts in object perception have been a major line of research. An important milestone along this line was set by Navon (1977), who, introducing his famous hierarchical letters stimulus and using the forest and trees metaphor, emphasized the distinction between global and local processing in the visual system (see also Navon, 2003). Since then, concepts such as global to local, global precedence (Navon, 1977; for a critical review, see Kimchi, 1992), reversed hierarchy (Hochstein \& Ahissar, 2002), and coarse to fine (Hughes, Nozawa, \& Kitterle, 1996; Loftus \& Harley, 2004; for a review, see Hegdé, 2008) have been used to describe the time course and the dynamics of visual perception. However, contemporary research has demonstrated that although global aspects of a visual scene are generally available before local details (e.g., Bar, 2004; see also Goffaux et al., 2011), the relative importance of using global and local visual information for object recognition is actually modulated by factors such as visual expertise (e.g., Curby, Glazek, \& Gauthier, 2009; Wong, Palmeri, \& Gauthier, 2009), task (e.g., Morrison \& Schyns, 2001), and the perceptual category of the perceived stimulus (e.g., McKone, Kanwisher, \& Duchaine, 2007). Therefore, recent studies have focused on the circumstances that can bias the usage of global or local information during visual perception. Within this framework, several studies have demonstrated global- or local-levelspecific priming effects (Forster, 2009; Hubner, 2000; Large \& McMullen, 2006; Robertson, 1996; Robertson, Egly, Lamb, \& Kerth, 1993; Shedden, Marsman, Paul, \& Nelson, 2003; Ward, 1982). Level-specific priming effects 
are interesting because, beyond the question of whether perceptual processing preferences can be induced top down and maintained across time, the outcome of such priming sheds light on the level of processing that is appropriate for a particular stimulus while a particular task is performed. A striking example of such research is the application of level-of-processing priming in studies of face perception.

It is widely acknowledged that human faces are processed holistically, in the sense that the identification of one part is influenced by the identity of other parts (Tanaka \& Farah, 1993; Young, Hellawell, \& Hay, 1987; for a review, see Farah, Wilson, Drain, \& Tanaka, 1998). Although faces are distinguished at the individual level by the relative spatial position of the inner components (second-order relations; Maurer, Grand, \& Mondloch, 2002), there is evidence that the processing of a spatial configuration is also affected by the global facial structure (Sergent, 1984; Tanaka \& Sengco, 1997). Assuming that global processing is a prerequisite of holistic perception, it has been hypothesized that face recognition should be impaired by priming local processes and might benefit from priming global processes. This hypothesis has received some empirical support.

For instance, in what could be considered as a seminal study, Macrae and Lewis (2002) reported that the ability of participants to identify a face among seven other faces as a previously seen individual was lower, relative to a control group, if, between study and test, participants spent $10 \mathrm{~min}$ attending to the local level of Navon stimuli. In contrast, participants who attended to the global level of the same stimuli during the study-test interval recognized the target face better than did the control group. This pattern has been replicated in several other studies (Hills \& Lewis, 2007; Lewis, Mills, Hills, \& Weston, 2009; Perfect, 2003; Perfect, Weston, Dennis, \& Snell, 2008; Weston, Perfect, Schooler, \& Dennis, 2008; see, however, Lawson, 2007). The link between global or local processing and holistic processing of faces has also been supported by a study in which participants were presented with composite faces (in which the top and the bottom halves were of different faces) following a Navon-stimulus-processing phase. In that study, the participants learned a set of four intact faces at the beginning of the experiment and selected, between two composite faces, the one that included either the eyes or the mouth of a previously learned face (Weston \& Perfect, 2005). The results showed that processing Navon stimuli for $3 \mathrm{~min}$ at the local level speeded the recognition of the components, suggesting that a local-processing strategy induced by priming helped overcome the detrimental effect of holistically processing the composite face (cf. Young et al., 1987). These data were presumed to support the transfer of processing-level strategies from the Navon to the composite-face task. However, this conclusion should be qualified because, while affecting response speed, the Navon task did not influence recognition accuracy for the components. Furthermore, processing the Navon stimuli at the global level did not interfere with recognition of the components in the face composites, albeit the participants were actually explicitly instructed to attend to the local level. In fact, in a subsequent study, Perfect et al. refined this interpretation, suggesting that the effect of processing the Navon stimuli on face recognition does not reflect transfer of either global- or local-level processing but, rather, whether the mode of processing the face, automatic or controlled, corresponds with the mode of processing the Navon stimuli. Specifically, matching only the upper half of composite faces requires the application of a controlled mechanism that allows ignoring the bottom halves, overcoming the automatic tendency to integrate the faces. Furthermore, if the structure of the Navon stimuli induces global precedence, focusing on their local level also requires controlled processing in order to overcome the automatic tendency to perceive the stimuli globally. The application of control mechanisms when Navon stimuli are processed might facilitate the application of control mechanisms while the face composites are matched. Conversely, when Navon stimuli that induce global precedence are processed at the global level, the automatic strategy established at that time may interfere with the application of a control mechanism required for compositeface matching. Consequently, according to this interpretation, if the Navon stimuli are structured to induce global precedence, addressing their global level should reduce performance in the composite-face-matching task, whereas addressing their local level should improve performance in this task. Importantly, this account suggests a general effect of correspondence between the perceptual strategies required in subsequent tasks and has no specific implication for face recognition.

An additional caveat about whether processing composite faces tell us much about face perception strategies is that decisional factors are also involved in the composite-face illusion (Richler, Gauthier, Wenger, \& Palmeri, 2008). By extension, it is possible that the priming level might also bias the decision criteria, rather than perceptual strategies. However, even assuming that the composite-face illusion reflects the joint effect of perceptual and decisional factors (see also Cheung, Richler, Palmeri, \& Gauthier, 2008), and although the magnitude of this effect is not necessarily correlated with face identification accuracy (Konar, Bennett, \& Sekuler, 2010), there is no argument that this illusion results from the tendency to process faces holistically (e.g., de Heering, Houthuys, \& Rossion, 2007; Grand, Mondloch, Maurer, \& Brent, 2004; Hole, 1994; Michel, Rossion, Han, Chung, \& Caldara, 2006; Rossion \& Boremanse, 2008; Schiltz, Dricot, Goebel, \& Rossion, 2010; Young et al., 
1987). Therefore, it is important to disentangle the possible effects of Navon priming on decisional and perceptual factors affecting the processing of composite faces. To address this challenge, in the present study, we adopted the full composite-face design (Gauthier \& Bukach, 2007; Richler, Tanaka, Brown, \& Gauthier, 2008) and took a signal detection approach, which allowed us to analyze the priming effect on decisional bias, as well as on perception sensitivity. On each trial, participants first matched two simultaneously presented Navon stimuli and then matched the upper halves of two sequentially presented composite faces. Navon matching required attention to either the global or the local level in separate blocks. Within each block, the composite faces were presented both aligned and misaligned. In the congruent conditions, the upper and the bottom halves of the two composite faces were either the same or different, whereas in the incongruent conditions, the upper and bottom halves lead to different responses. To probe whether priming local processing facilitates processing the two halves of the composite face independently, whereas priming global processing encourages integration and, consequently, interferes with the independent processing of each halfface, we compared the congruency effect (congruent incongruent) in each priming condition with a baseline. This baseline was contributed by a different group of participants who performed the composite-face task without any priming. We predicted smaller congruency effects following the local than following the global priming if the half-faces in the composite were aligned but no such effects if they were misaligned. If both types of processing-level priming affect sensitivity to the congruence between the two halves of the composite faces (in opposite ways), the congruency effect should be larger following global priming, relative to baseline, but smaller, relative to baseline, following local priming. Finally, if priming affects decision-making processes, we should find priming effects on the response criterion.

\section{Method \\ Participants}

Twenty-four undergraduates from the Hebrew University (mean age $22.8 \pm 2.9$ years; 17 females) were tested in the priming conditions, and 17 naïve participants (mean age $22.2 \pm 4.2$ years; 10 females) were tested in the baseline condition. One female participant in the baseline group was excluded because of overall low accuracy. All the participants had normal or corrected-to-normal visual acuity and no history of psychiatric or neurological disorders. Participants signed an informed consent form according to the requirements of the institutional review board of the Hebrew University and were paid for participation or received course credit.

Stimuli, task, and design

The Navon stimuli consisted of black letters presented on a gray background. Each local letter was $0.85 \mathrm{~cm}$ wide $\times 1.14 \mathrm{~cm}$ high $\left(0.7^{\circ} \times 0.9^{\circ}\right)$; the local letters were spatially arranged on a $5 \times 5$ grid to form a global letter that was $5.8 \mathrm{~cm}$ wide $\times 6 \mathrm{~cm}$ high $\left(4.7^{\circ} \times 4.9^{\circ}\right)$. The letters were black Helvetica bold font D, E, F, and $\mathrm{H}$ in all their local and global combinations, making 16 distinct Navon stimuli (Fig. 1a).

Face stimuli were generated from 96 grayscale images of unfamiliar Caucasian faces, half males and half females. The original images were first cut to form an ellipse shape $5.30 \mathrm{~cm}$ wide $\times 7.40 \mathrm{~cm}$ high $\left(4.3^{\circ} \times 6.0^{\circ}\right)$ that excluded exterior face features. To create composite faces, we divided the vertical dimension of each face into two halves. The top and the bottom parts from same-gender faces were recombined, leaving a $0.19-\mathrm{cm}$ gap between the two parts. The top half of each face was recombined with its original bottom half, as well as with the bottom half of a different face chosen at random without replacement. This resulted in 384 different composite faces in total. Right shifting the bottom part of the aligned faces $2.65 \mathrm{~cm}$, we produced 384 misaligned faces (Fig. 1b). Each composite face was used only once in the experiment.

In the priming task, two Navon stimuli were presented simultaneously, one in the left and one in the right visual field, each with the most medial edge $0.25^{\circ}$ from fixation. The participants were instructed to match the two displays, focusing either on the large letters, ignoring the small letters (global level), or on the small letters, ignoring the large letters (local level).

a

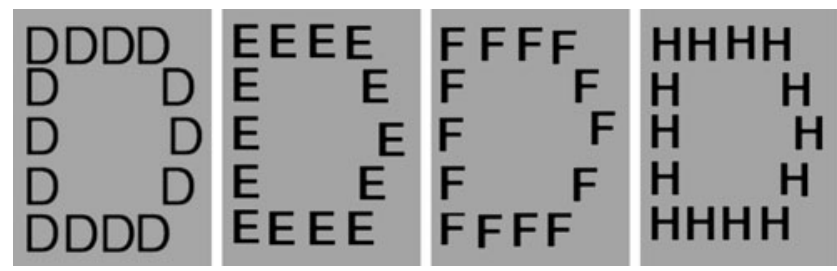

b

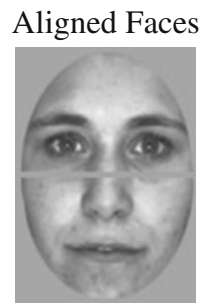

Misaligned Faces

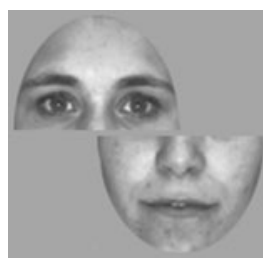

Fig. 1 Examples of a Navon letters used in the present study and $\mathbf{b}$ aligned and misaligned composite faces used in the present study 
The global and the local prime processing tasks were blocked, and the order of blocks was counterbalanced across participants.

In the composite-face task, two composite faces were presented one after the other at fixation, and the participants were instructed to match the top halves of the two composites, ignoring the bottom halves. The first face on each trial was composed from the top and the bottom halves of the same face, while the combination of the second composite face defined one of four pair-type conditions, two congruent and two incongruent. In the congruent pairs, the top and the bottom halves of the two composites were taken either from the same faces or from different faces: top-same and bottom-same or topdifferent and bottom-different faces. In the incongruent pairs, either the top or the bottom half was taken from the same face, while the other halves were taken from different faces: top-same and bottom-different or topdifferent and bottom-same faces (Fig. 2). For the priming group, these four types of composite faces were presented aligned and misaligned to each participant. The eight composite-face task conditions were mixed and presented in random order within the global- and the local-priming blocks. The baseline group was tested only with aligned stimuli. The same four types of composite faces were randomized in one block.

\section{Procedure}

The stimuli were shown on a 17 -in. CRT monitor with a vertical refresh rate of $100 \mathrm{~Hz}$ and a resolution of $1,024 \times 768$ pixels. Participants were seated $70 \mathrm{~cm}$ from the screen in a dimly lit room. Trial timing was controlled by Presentation (Neurobehavioral Systems, Albany, CA), as depicted in Fig. 3.

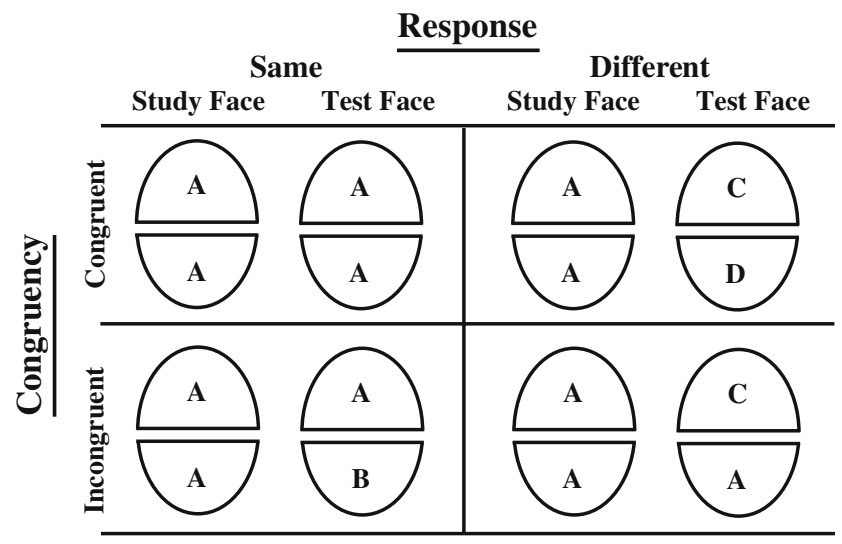

Fig. 2 Illustration of the conditions adopted in the composite paradigm for the aligned conditions. Different letters represent different identities
For the experimental group, each trial began with a red fixation mark that appeared at the center of the screen for $500 \mathrm{~ms}$. The fixation mark was followed by the two Navon stimuli, to which the participants responded by pressing either a "match" or a "mismatch" key with their nondominant hand. The Navon stimuli were presented until response or for up to $2 \mathrm{~s}$. The offset of the Navon stimuli was followed by a 400-ms blank interval, after which the first of the two composite faces was displayed for $250 \mathrm{~ms}$. The second composite face was displayed after an additional blank interval of $250 \mathrm{~ms}$ and was exposed until a response or for up to $2 \mathrm{~s}$. The participants responded to the composite-face task using their dominant hand, selecting between a "same" or a "different" key. Accuracy, rather than speed, was emphasized. Following a blank intertrial interval of $700-900 \mathrm{~ms}$, a new trial began automatically.

For the baseline group, the procedure was identical, except that the stimulus onset asynchrony (SOA) between the fixation cross and the first composite face was $1,300 \mathrm{~ms}$ (roughly equal to the SOA between the Navon and the first composite face in the priming conditions) and the red fixation was also presented during the blank interval between the two composite faces.

There were 48 trials in each condition, with a total of 384 trials per priming-level block. Each experimental block was preceded by 32 practice trials. Each block was split into four sessions of 96 trials each, with a break of $5 \mathrm{~min}$ between sessions. The entire experiment lasted approximately $50 \mathrm{~min}$ for the priming group and about $15 \mathrm{~min}$ for the baseline group.

\section{Results}

We first analyzed the performance of the priming group with a within-subjects ANOVA. The factors were priming level (global, local) $\times$ alignment (aligned, misaligned) $\times$ congruency (congruent, incongruent).

Performance in the Navon priming task

The structure of the Navon stimuli led to the frequently observed global precedence. Matching the Navon letters was more accurate and faster at the global level than at the local level, and the difference was not influenced by the congruency or the alignment of the subsequent composite faces. An ANOVA showed a significant main effect of priming level for accuracy $(M$ global $=95 \% ; M$ local $=93 \%), F(1,23)=10.5$, $M S E=0.002, p<.005$, partial $\eta^{2}=.3$, as well as for response time (RT; $M$ global $=830 \mathrm{~ms} ; M$ local $=894 \mathrm{~ms}$ ), $F(1,23)=7.7, M S E=5,606, p<.025$, partial $\eta^{2}=.2$. There were no other significant main effects or interactions (all type I error probabilities $>.25$ ). 
Fig. 3 Illustration of the time course of a trial in the present study

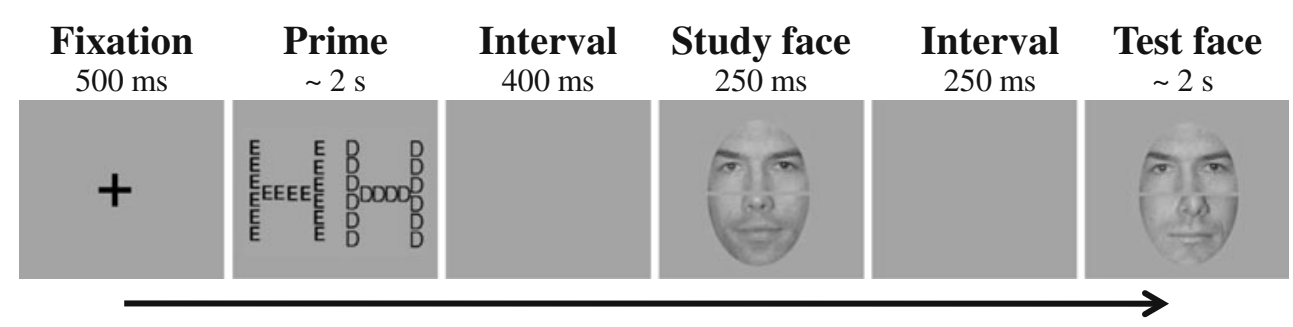

Performance in the composite-face task

Only trials on which the response to the prime was correct (94\% of the trials) were included in the composite-face task analysis. Sensitivity to face identity was determined by $\mathrm{A}_{z}$, which is a monotonic transformation of $d$ ' bounded between 0 and 1. Previous studies suggested that the $A_{z}$ is more robust than $d$ ' to the influence of response bias (Richler, Tanaka, et al., 2008; Verde, Macmillan, \& Rotello, 2006).

As is shown in Fig. 4a, in the aligned condition, the processing level of Navon letters influenced the participants' responses to faces, with the lowest sensitivity in the globalincongruent condition. No priming effects were found in any other condition.

An ANOVA showed that the sensitivity was lower when the composite faces were aligned $\left(A_{z}=.95\right)$ than when they were misaligned $\left(A_{z}=.97\right), F(1,23)=17.3, M S E=0.001$, $p<.001$, partial $\eta^{2}=.4$, and was lower in the incongruent condition $\left(A_{z}=.95\right)$ than in the congruent condition $\left(A_{z}=\right.$ .97), $F(1,23)=13.6, M S E=0.001, p<.001$, partial $\eta^{2}=$ .4. The alignment $\times$ congruency interaction was also significant, $F(1,23)=40.3, M S E=0.001, p<.001$, partial $\eta^{2}=.6$, indicating that congruency affected sensitivity only in the aligned condition, $t(23)=5.58$, $p<.001$, yet there was no trend for congruency effect in the misaligned condition, $t(23)<1$. This pattern is the hallmark of holistic processing of faces.

Importantly, the second-order priming level $\times$ alignment $\times$ congruency interaction was significant, $F(1,23)=5.1, M S E=$ $0.001, p<.05$, partial $\eta^{2}=.2$, suggesting that the priming level $\times$ congruency interaction was different for aligned and misaligned composite faces. This interaction was investigated with separate two-way ANOVAs for the aligned and misaligned conditions.

These analyses showed that the congruency $\times$ priming level interaction was significant for the aligned condition, $F(1,23)=6.7, M S E=0.001, p<.025$, partial $\eta^{2}=.2$, but not for the misaligned condition, $F(1,23)<1$. A post hoc exploration of the congruency $\times$ priming level interaction for the aligned composite faces revealed that the congruency effect in the aligned condition was significant both in the global-priming condition (.98 vs. .92), $t(23)=5.20, p<$ .001 , and in the local-priming condition (.98 vs. .95$), t(23)=$
$5.20, p<.0025$. This suggests that even after local priming, there was a tendency to process the composite faces holistically. However, as was predicted, the congruency effect was significantly lower after local priming than after global priming (.03 vs. .06), $t(23)=2.60, p<.025$. There were no other significant effects (all type I error probabilities $>.25$ ).

The analysis of the response criterion was based on a similar ANOVA design (Fig. 4b). This analysis showed that the response bias toward different decisions was larger in the incongruent $(c=.8)$ than in the congruent $(c=.01)$ condition, $F(1,23)=17.6, M S E=0.02, p<.001$, partial $\eta^{2}=.4$, and larger for aligned $(c=.8)$ than for misaligned $(c=.01)$ composite faces, $F(1,23)=19.6, M S E=0.01, p<$ .001 , partial $\eta^{2}=.5$. The congruency $\times$ alignment interaction was significant, $F(1,23)=8.0 M S E=0.02, p<.01$, partial $\eta^{2}=.3$, indicating that the congruency effect was significant in the aligned condition, $t(23)=4.19, p<.001$, but not in the misaligned condition, $t(23)=1.24, p=.23$. The most important result of this analysis, however, was that there was no priming-level effect overall, $F(1,23)<1$, and no priming level $\times$ alignment interaction, $F(1,23)<1$. There was a tendency for a priming level $\times$ congruency interaction, $F(1,23)=4.2, M S E=0.008, p=.051$, partial $\eta^{2}=.16$, which did not further interact with alignment, $F(1,23)=$ $1.9, M S E=0.011, p=.18$, partial $\eta^{2}=.07$. Post hoc comparisons across alignment showed that the bias toward different responses was slightly larger in the local- than in the global-priming conditions for congruent composite faces (mean difference $=.014$ ) and slightly smaller in the local- than in the global-priming conditions for incongruent composite faces (mean difference $=-.04$ ). However, none of these differences were significant, $t(23)<1.0$ and $t(23)=-1.46, p=.157$, for the congruent and incongruent conditions, respectively. Finally, notwithstanding the absence of a second-order interaction, since the congruency effect on $A_{z}$ was significant only in the aligned condition, we compared the congruency effect on the criterion in the global and local conditions, focusing only on aligned composites. This analysis showed that the congruency effect was similar for the global-priming (-.13) and local-priming $(-.12)$ conditions. In other words, the source of the priming level $\times$ congruency interaction was in 
a

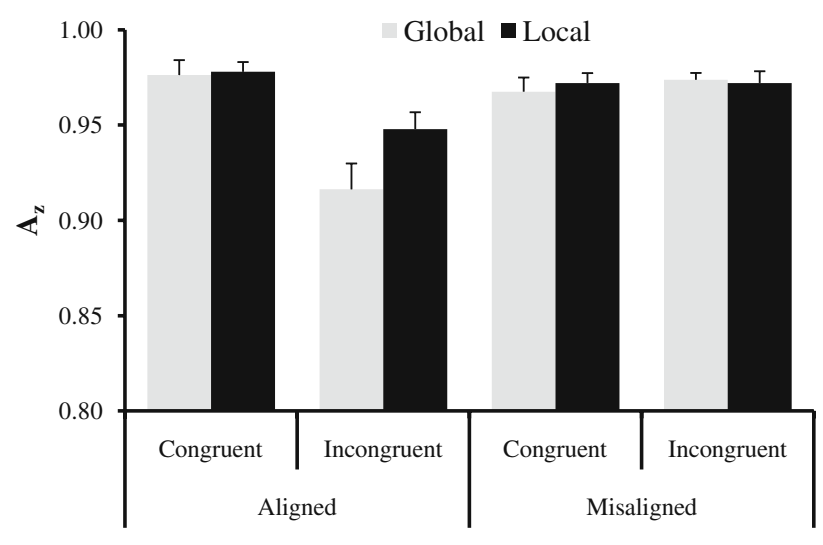

b

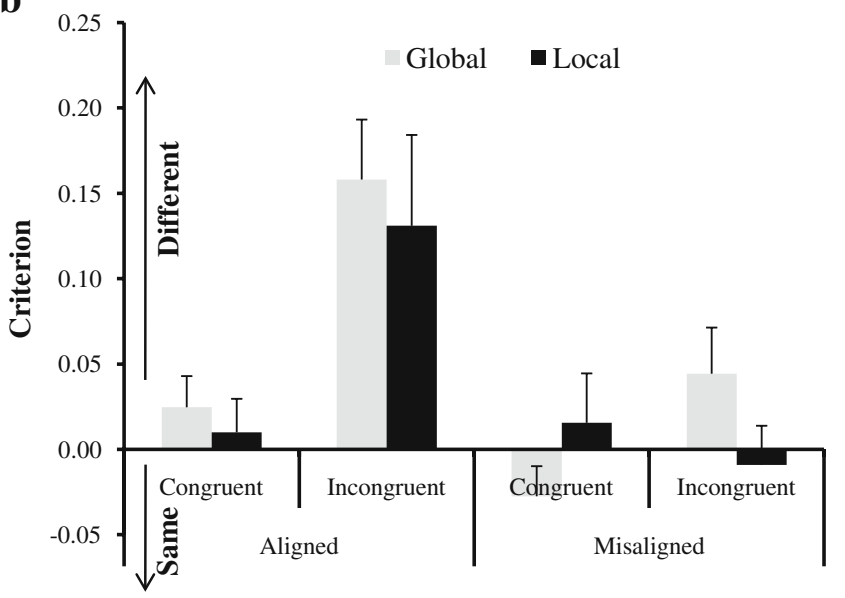

Fig. 4 Mean $\mathbf{a} A_{z}$ and $\mathbf{b}$ criterion for the composite-face task adopting the complete design calculation. Error bars show standard errors

the misaligned condition, where $A_{z}$ was not influenced by the priming level. ${ }^{1}$

\section{Comparison with baseline}

Since the priming level was nested only in the experimental group, to assess the effects of global and local priming relative to baseline, we compared the congruency effect for the experimental group with that for the control group separately for each priming condition. These comparisons were based on mixed-model ANOVAs with group (experimental, control) as the between-subjects factor and congruency (congruent, incongruent) as a within-subjects factor.

As is shown in Fig. 5a, across the global-, local-, and nopriming conditions, $A_{z}$ was consistently higher in the congruent than in the incongruent condition. Confirming this observation, the mixed ANOVA revealed higher sensitivity in the congruent than in the incongruent condition across groups in both priming conditions, $F(1,38)=25.6$, $M S E=0.001, p<.001$, partial $\eta^{2}=.40$ for global priming, and $F(1,38)=15.6, M S E=0.001, p<.001$, partial $\eta^{2}=.29$ for local priming. However, whereas for the global-priming condition there was a significant congruency $\times$ group interaction, $F(1,38)=11.7, M S E=0.001, p<.001$, partial

\footnotetext{
${ }^{1}$ Since we explicitly emphasized accuracy at the expense of speed, the RTs were not very informative. Nevertheless, we analyzed the RT data using the same design as for $A_{z}$. This analysis revealed that in the aligned condition for both global and local priming, the RTs were significantly longer in the incongruent condition than in the congruent condition. There were no significant RT effects in the misaligned conditions. Moreover, there was no speed-accuracy trade-off in the composite-face task (the full analysis is available as supplementary materials).
}

$\eta^{2}=.24$, this interaction was not significant for the localpriming condition, $F(1,38)=3.1, M S E=0.001, p=.087$; partial $\eta^{2}=.07$. The analysis of the two-way interaction in the global condition showed that the congruency effect was significantly larger in the global-priming condition than in the baseline (no-priming) condition (0.06), $t(38)=4.138, p<.001$, but the latter was still significant (as compared with zero), $\mathrm{t}(15)=3.897, p<.001$. Finally, overall, sensitivity was higher in the baseline than in both experimental conditions, $F(1,38)=14.1, M S E=0.003$, $p<.001$, partial $\eta^{2}=.27$, and $F(1,38)=13.2, M S E=$ $0.001, p<.001$, partial $\eta^{2}=.26$, for the global- and localpriming conditions, respectively, suggesting an overall effect of interference by the Navon task on the composite-face task.

A similar analysis was conducted on criterion (see Fig. 5b).The mixed ANOVA showed that the response bias towards different decisions was larger in the incongruent than in the congruent condition across groups, $F(1,38)=$ 21.733, MSE $=0.232, p<.001$, partial $\eta^{2}=.36$ for the comparison with global priming, and $F(1,38)=12.62, M S E=$ $0.206, p<.001$, partial $\eta^{2}=.25$ for the comparison with local priming. Critically, there was no congruency $\times$ group interaction for both priming levels (all type I error probabilities $>.30$, suggesting that the difference of congruency effect revealed in $A_{z}$ was not influenced by the response criterion.

\section{Discussion}

The results of the present study revealed that the compositeface illusion, as reflected by sensitivity to the congruency between the upper and the lower halves of composite faces, was enhanced when participants attended to the global level 
a

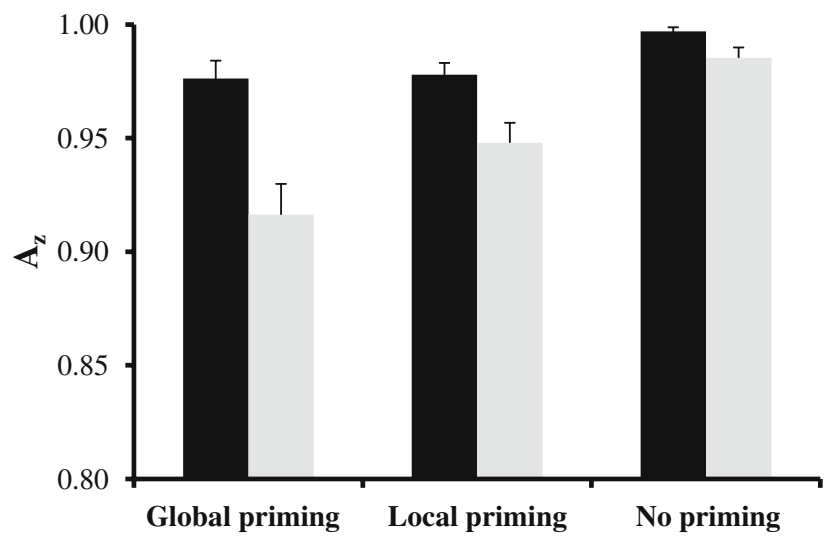

b

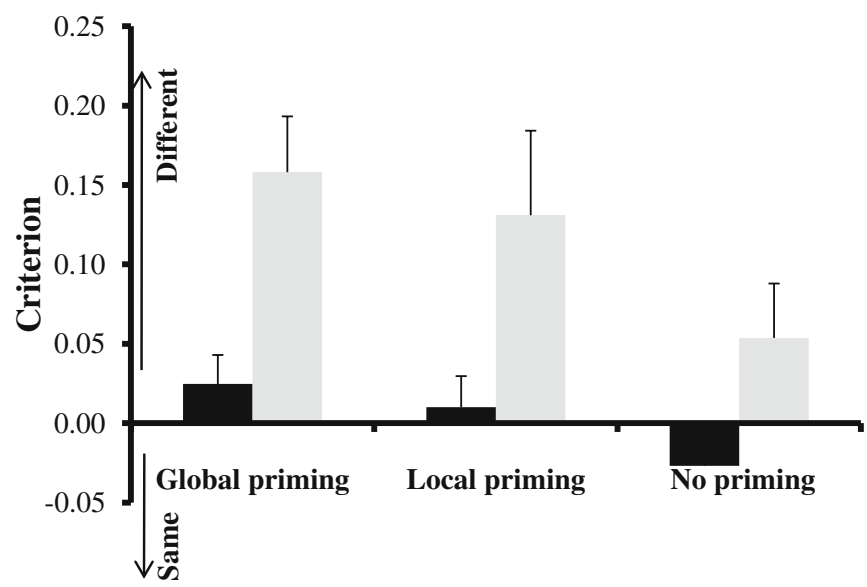

Congruent

Incongruent

Fig. 5 Congruency effects following global, local, or no priming and the respective response criteria

of Navon stimuli prior to face processing, yet attending to the local level was inconsequential.

To reiterate, the composite-face illusion occurs while the participant attempts to match two identical halves of the same face, each of which is combined with a different bottom half. Since its initial demonstration (Young et al., 1987), numerous studies have replicated the basic effect, showing that the percentage of (correct) same responses is considerably lower when the top- and the bottom-half faces are aligned than when the two halves of each composite are misaligned. This effect has frequently been attributed to the unavoidable integration of the bottom half with the top half of each face, and therefore, the composite-face illusion has been considered a hallmark of holistic face processing (cf. Tanaka \& Farah, 1993). This account has also been supported by recent ERP findings (Jacques \& Rossion, 2009, 2010; Kuefner, Jacques, Prieto, \& Rossion, 2010; Schiltz et al., 2010). To this end, the pattern of priming effects in our present study suggests that allocating attention to the global level of preceding Navon stimuli augments the tendency to process faces holistically.

Two important aspects of our present design support this conclusion. First, using the complete design of the composite-face matching task (Richler, Gauthier, et al., 2008), we were able to attribute the priming effect induced by the processing of Navon stimuli to perceptual aspects of face processing. Sensitivity to the congruency of the composite faces in a pair was evident only in the aligned condition, where the detrimental effect of incongruence on accuracy was enhanced by priming global perception and unaffected by priming local perception. In contrast, the analysis of the criterion showed that although there was a response bias to select the different response in the critical aligned/incongruent condition, ${ }^{2}$ this bias was not modulated by the priming level. Hence, whereas our findings support the involvement of a response bias factor in composite-facematching performance (Cheung et al., 2008), processinglevel priming does not affect this factor. This is, to our knowledge, the first direct evidence that Navon priming affects the perceptual, rather the response/decisional, level.

Second, the present trial-by-trial priming design provides a more direct test of global- and local-priming effects on holistic and analytic processing of faces. In most previous studies, the Navon stimuli were introduced between the study and test phases. Hence, even when composite faces were used as targets, processing the study face was hardly influenced by the processing level of the Navon stimuli. An exception to this rule is a recent study by Lewis et al. (2009). In that study, participants learned a set of unfamiliar faces and were tested for subsequent recognition. Prior to learning, as well as prior to the test phase, participants were presented three Navon stimuli and were instructed to name either the global letter or the local letters. The most important result in this study was that processing the Navon stimuli at the same level prior to study and prior to test improved face recognition, relative to conditions in which the required processing level of the Navon stimuli was different between study and test. Interestingly this improvement was observed regardless of the level at which the Navon stimuli were processed.

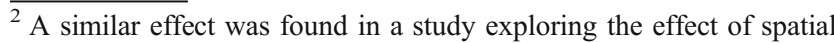
frequency ranges on the composite effect (Cheung et al., 2008).
} 
This pattern suggests that the level of processing induced during the Navon task determines the kind of information that is encoded in episodic memory (cf. transfer of appropriate processing; Morris, Bransford, \& Franks, 1977). However, additional evidence was necessary to conclude that perceptual (distinct from memory) processes are affected by the level at which the Navon letters are identified. Such evidence was provided by the present design, which predominantly invoked perception, rather than memory, and both the first and the second composite faces were presented after global or local processing of the Navon stimuli. Furthermore, the composite-face illusion more clearly reflects holistic processes than does simple face recognition, which, as was suggested by the study of Lewis and colleagues, may involve both featural and configural, as well as holistic, processes (Maurer et al., 2002).

Intriguingly, the present findings are different than those reported by Weston and Perfect (2005) in two ways. First, whereas in that previous study, local priming facilitated performance and global priming was inconsequential, in our present study, the opposite was true: Global priming interfered with performance, whereas local priming was inconsequential. Second, whereas the priming in Weston and Perfect's study affected speed but not accuracy, here we found effects on accuracy, whereas for RTs the effects were less convincing. ${ }^{3}$ Some of these differences could probably be explained by the difference between the two task settings. As we noted, the specific instructions that the participants received in the former study were to attend to particular components (either the eyes or the mouth). This task might have induced a much stronger tendency for local processing than did our half-face matching task. This could explain why local-level priming facilitated performance in the former study, but not in ours. Along the same line, it is possible that the explicit requirement to allocate attention to a face component could reduce the default holistic-face-processing strategy, or even eliminate it. Hence, priming global-level processing might have not been effective. On the other hand, since in our present study the composite faces were presented one at a time and the participants needed to pay attention to the entire upper part of the face, priming local processing might have not helped much, while priming global processing could have enhanced the normal tendency to integrate the two halves of the face into one whole. Whereas this interpretation stands to reason, we should consider it with caution because, as compared with baseline, even following local priming the congruency effect tended to

\footnotetext{
$\overline{3}$ Overall, as was expected, the responses were slower to incongruent composite faces than to congruent ones in the aligned, but not in the misaligned, condition. However, the difference between global and local priming in the aligned condition was not significant in either the congruent or the incongruent condition (see supplementary materials).
}

be larger in the priming group than in the experimental group. Although this tendency was insufficient to lend a significant interaction between congruency and group, the numerically higher effects in the local-priming condition than in the baseline condition were contrary to what we expected and are intriguing. Note, however, that this pattern cannot be explained by Perfect et al.'s (2008) hypothesis as well. Since the structure of the presently used Navon stimuli induced global precedence (Flevaris, Bentin, \& Robertson, in press), processing the local level should have triggered a control mechanism that, according to Perfect and colleagues, was supposed to reduce the congruency effect, relative to baseline. Finally, regarding the RTs, perhaps the fuzzy pattern reflects the fact that we explicitly emphasized accuracy at the expense of speed.

While suggesting that the present priming effects reflect the modulation of holistic face processing, lower level vision factors that might affect performance should also be considered. One is that, in the local priming condition, participants might have adopted the strategy of foveating local letters in the upper half of the global letter and continued fixating the upper part of the visual field while processing the composite faces. This strategy might explain the smaller congruency effect in the local-priming conditions. Since we did not monitor eye movements, we cannot exclude this possibility. However, we consider this account unlikely, because the Navon stimuli were always preceded by a fixation mark and were vertically centered with respect to this mark, while the monitor was elevated to the participants' eye level. Moreover, in the baseline experiment, the fixation mark was continuously displayed during the interstimulus interval between the two faces, as well as preceding the first face composite. Nevertheless, the more stringent control of eye movements in the baseline condition did not much change the congruency effect, relative to the local-priming condition in which the fixation mark was presented only prior to the Navon stimuli.

Another possible interpretation is that the level of processing of the Navon stimuli (global or local) does not influence directly the face-processing strategy (holistic or component based). Rather, this influence is mediated by a biased selection of spatial frequencies (SFs) during visual perception (Hills \& Lewis, 2009; see also Weston \& Perfect, 2005). Evidence that processing Navon stimuli can bias the selection of SFs in a subsequent image has recently been provided by a study that demonstrated that allocating attention to the global or local levels of Navon stimuli facilitates the processing of low SFs or high SFs, respectively, in subsequently presented compound-frequency gratings (Flevaris et al., 2010, in press). Evidence that holistic processing relies primarily on LSFs, while component-based processing relies primarily on high SFs, has also been provided (e.g., 
Goffaux, 2009; Goffaux \& Rossion, 2006; Robertson, 1996; Shulman, Sullivan, Gish, \& Sakoda, 1986). Hence, it is possible that biasing the selection of SFs in the image is the mechanism by which global processing facilitates holistic processing. Furthermore, in a recent study, we demonstrated that the accumulation of LSFs in visual short-term memory (VSTM) is prioritized (Gao \& Bentin, in press). Similarly, Goffaux et al. (2011) showed that LSFs are also prioritized in the perception of faces. Since, in the present study, the two composite faces were presented in sequence with a 500-ms SOA, the prioritized accumulation of LSFs in both perception and VSTM might partly explain why we found effects following global priming, but not following local priming.

In conclusion, the present study allowed teasing apart the influences of global and local priming on perceptual and decisional factors that contribute to the composite-face illusion. Specifically, we showed that priming global-level processing augments the tendency to integrate the two halffaces and increases the sensitivity to the congruency between them. In contrast, although a response bias factor contributes to the composite-face illusion, it is not modulated by priming. This outcome provides additional evidence for the traditional argument that holistic perception is an important factor inducing the composite-face illusion and, by extension, that faces are processed holistically by default (Young et al., 1987).

Acknowledgement This study was funded by NIMH Grant R01 MH 64458 to Lynn Robertson and Shlomo Bentin. Zaifeng Gao was partially supported by the Lady Davis Fellowship. We are grateful to Alaa Watad, Noa Gazit, and Raanan Greenman for skillful research assistance. Lynn C. Robertson has a Senior Research Career Scientist award from the Veterans Administration and is affiliated with the VA Clinical Sciences Research Service, Department of Veterans Affairs Medical Center, Martinez, CA.

Open Access This article is distributed under the terms of the Creative Commons Attribution Noncommercial License which permits any noncommercial use, distribution, and reproduction in any medium, provided the original author(s) and source are credited.

\section{References}

Bar, M. (2004). Visual objects in context. Nature Reviews. Neuroscience, $5,617-629$.

Cheung, O. S., Richler, J. J., Palmeri, T. J., \& Gauthier, I. (2008). Revisiting the role of spatial frequencies in the holistic processing of faces. Journal of Experimental Psychology: Human Perception and Performance, 34, 1327-1336.

Curby, K. M., Glazek, K., \& Gauthier, I. (2009). A visual short-term memory advantage for objects of expertise. Journal of Experimental Psychology: Human Perception and Performance, 35, 94-107.

de Heering, A., Houthuys, S., \& Rossion, B. (2007). Holistic face processing is mature at 4 years of age: Evidence from the composite face effect. Journal of Experimental Child Psychology, 96, 57-70.
Farah, M. J., Wilson, K. D., Drain, M., \& Tanaka, J. N. (1998). What is "special" about face perception? Psychological Review, 105, $482-498$.

Flevaris, A. V., Bentin, S., \& Robertson, L. C. (2010). Local or global? Attentional selection of spatial frequencies binds shapes to hierarchical levels. Psychological Science, 21, 424-431.

Flevaris, A. V., Bentin, S., \& Robertson, L. (in press). Attention to hierarchical level influences attentional selection of spatial scale. Journal of Experimental Psychology: Human Perception and Performance

Forster, J. (2009). Relations between perceptual and conceptual scope: How global versus local processing fits a focus on similarity versus dissimilarity. Journal of Experimental Psychology: General, 138, 88-111.

Gao, Z., \& Bentin, S. (in press). Coarse-to-fine encoding and impartial decay of spatial frequency information into visual short-term memory for faces. Journal of Experimental Psychology: Human Perception and Performance

Gauthier, I., \& Bukach, C. (2007). Should we reject the expertise hypothesis? Cognition, 103, 322-330.

Goffaux, V. (2009). Spatial interactions in upright and inverted faces: Re-exploration of spatial scale influence. Vision Research, 49, 774-781.

Goffaux, V., Peters, J., Haubrechts, J., Schiltz, C., Jansma, B., \& Goebel, R. (2011). From coarse to fine? Spatial and temporal dynamics of cortical face processing. Cerebral Cortex, 21, 467476. doi:10.1093/cercor/bhq112

Goffaux, V., \& Rossion, B. (2006). Faces are "spatial"-Holistic face perception is supported by low spatial frequencies. Journal of Experimental Psychology: Human Perception and Performance, 32, 1023-1039.

Grand, R. L., Mondloch, C. J., Maurer, D., \& Brent, H. P. (2004). Impairment in holistic face processing following early visual deprivation. Psychological Science, 15, 762-768.

Hegdé, J. (2008). Time course of visual perception: Coarse-to-fine processing and beyond. Progress in Neurobiology, 84, 405439.

Hills, P. J., \& Lewis, M. B. (2007). Temporal limitation of Navon effect on face recognition. Perceptual and Motor Skills, 104, 501-509.

Hills, P. J., \& Lewis, M. B. (2009). A spatial frequency account of the detriment that local processing of Navon letters has on face recognition. Journal of Experimental Psychology: Human Perception and Performance, 35, 1427-1442.

Hochstein, S., \& Ahissar, M. (2002). View from the top: Hierarchies and reverse hierarchies in the visual system. Neuron, 36, 791804.

Hole, G. J. (1994). Configurations! Factors in the perception of unfamiliar faces. Perception, 23, 65-74.

Hubner, R. (2000). Attention shifting between global and local target levels: The persistence of level-repetition effects. Visual Cognition, 7, 465-484.

Hughes, H. C., Nozawa, G., \& Kitterle, F. (1996). Global precedence, spatial frequency channels, and the statistics of natural images. Journal of Cognitive Neuroscience, 8, 197-230.

Jacques, C., \& Rossion, B. (2009). The initial representation of individual faces in the right occipito-temporal cortex is holistic: Electrophysiological evidence from the composite face illusion. Journal of Vision, 9(6, Art. 8), 1-16.

Jacques, C., \& Rossion, B. (2010). Misaligning face halves increases and delays the N170 specifically for upright faces: Implications for the nature of early face representations. Brain Research, $1318,96-109$.

Kimchi, R. (1992). Primacy of wholistic processing and global local paradigm: A critical review. Psychological Bulletin, 112, 24-38. 
Konar, Y., Bennett, P. J., \& Sekuler, A. B. (2010). Holistic processing is not correlated with face-identification accuracy. Psychological Science, 21, 38-43.

Kuefner, D., Jacques, C., Prieto, E. A., \& Rossion, B. (2010). Electrophysiological correlates of the composite face illusion: Disentangling perceptual and decisional components of holistic face processing in the human brain. Brain and Cognition, 74, $225-238$.

Large, M. E., \& McMullen, P. A. (2006). Hierarchical attention in discriminating objects at different levels of specificity. Perception \& Psychophysics, 68, 845-860.

Lawson, R. (2007). Local and global processing biases fail to influence face, object, and word recognition. Visual Cognition, $15,710-740$

Lewis, M. B., Mills, C., Hills, P. J., \& Weston, N. (2009). Navon letters affect face learning and face retrieval. Experimental Psychology, 56, 258-264.

Loftus, G. R., \& Harley, E. A. (2004). How different spatial-frequency components contribute to visual information acquisition. Journal of Experimental Psychology: Human Perception and Performance, 30, 104-118.

Macrae, C. N., \& Lewis, H. L. (2002). Do I know you? Processing orientation and face recognition. Psychological Science, 13, 194196

Maurer, D., Grand, R. L., \& Mondloch, C. J. (2002). The many faces of configural processing. Trends in Cognitive Sciences, 6, 255-260.

McKone, E., Kanwisher, N., \& Duchaine, B. C. (2007). Can generic expertise explain special processing for faces? Trends in Cognitive Sciences, 11, 8-15.

Michel, C., Rossion, B., Han, J., Chung, C. S., \& Caldara, R. (2006). Holistic processing is finely tuned for faces of one's own race. Psychological Science, 17, 608-615.

Morris, C. D., Bransford, J. P., \& Franks, J. J. (1977). Levels of processing versus transfer appropriate processing. Journal of Verbal Learning and Verbal Behavior, 16, 519-533.

Morrison, D. J., \& Schyns, P. G. (2001). Usage of spatial scales for the categorization of faces, objects, and scenes. Psychonomic Bulletin \& Review, 8, 454-469.

Navon, D. (1977). Forest before trees: The precedence of global features in visual perception. Cognitive Psychology, 9, 353-383.

Navon, D. (2003). What does a compound letter tell the psychologist's mind? Acta Psychologica, 114, 273-309.

Perfect, T. J. (2003). Local processing bias impairs lineup performance. Psychological Reports, 93, 393-394.

Perfect, T. J., Weston, N. J., Dennis, I., \& Snell, A. (2008). The effects of precedence on Navon-induced processing bias in face recognition. The Quarterly Journal of Experimental Psychology, 61, 1479-1486.

Richler, J. J., Gauthier, I., Wenger, M. J., \& Palmeri, T. J. (2008). Holistic processing of faces: Perceptual and decisional components. Journal of Experimental Psychology. Learning, Memory, and Cognition, 34, 328-342.
Richler, J. J., Tanaka, J. W., Brown, D. D., \& Gauthier, I. (2008). Why does selective attention to parts fail in face processing? Journal of Experimental Psychology. Learning, Memory, and Cognition, 34, 1356-1368.

Robertson, L. C. (1996). Attentional persistence for features of hierarchical patterns. Journal of Experimental Psychology: General, 125, 227-249.

Robertson, L. C., Egly, R., Lamb, M. R., \& Kerth, L. (1993). Spatial attention and cuing to global and local levels of hierarchical structure. Journal of Experimental Psychology: Human Perception and Performance, 19, 471-487.

Rossion, B., \& Boremanse, A. (2008). Nonlinear relationship between holistic processing of individual faces and picture-plane rotation: Evidence from the face composite illusion. Journal of Vision, 8 (4, Art. 3), 1-13.

Schiltz, C., Dricot, L., Goebel, R., \& Rossion, B. (2010). Holistic perception of individual faces in the right middle fusiform gyrus as evidenced by the composite face illusion. Journal of Vision, 10 (2, Art. 25), 1-16.

Sergent, J. (1984). An investigation into component and configural processes underlying face perception. British Journal of Psychology, $75,221-242$

Shedden, J., Marsman, I., Paul, M., \& Nelson, A. (2003). Attention switching between global and local elements: Distracter category and the level repetition effect. Visual Cognition, 10, 433-470.

Shulman, G. L., Sullivan, M. A., Gish, K., \& Sakoda, W. J. (1986). The role of spatial-frequency channels in the perception of local and global structure. Perception, 15, 259-273.

Tanaka, J. W., \& Farah, M. J. (1993). Parts and wholes in face recognition. The Quarterly Journal of Experimental Psychology, $46 A, 225-245$.

Tanaka, J. W., \& Sengco, J. A. (1997). Features and their configuration in face recognition. Memory \& Cognition, 25, 583-592.

Verde, M. F., Macmillan, N. A., \& Rotello, C. M. (2006). Measures of sensitivity based on a single hit rate and false alarm rate: The accuracy, precision, and robustness of $d^{\prime}, A_{z}$, and $A^{\prime}$. Perception \& Psychophysics, 68, 643-654.

Ward, L. M. (1982). Determinants of attention to local and global features of visual forms. Journal of Experimental Psychology: Human Perception and Performance, 8, 562-581.

Weston, N. J., \& Perfect, T. J. (2005). Effects of processing bias on the recognition of composite face halves. Psychonomic Bulletin \& Review, 12, 1038-1042.

Weston, N. J., Perfect, T. J., Schooler, J. W., \& Dennis, I. (2008). Navon processing and verbalisation: A holistic/featural distinction. European Journal of Cognitive Psychology, 20, 587-611.

Wong, A. C. N., Palmeri, T. J., \& Gauthier, I. (2009). Conditions for facelike expertise with objects: Becoming a ziggerin expert-but which type? Psychological Science, 20, 1108-1117.

Young, A. W., Hellawell, D., \& Hay, D. C. (1987). Configurational information in face perception. Perception, 16, 747-759. 